\title{
Predictive effects of preoperative serum CA125 and AFP levels on post-hepatectomy survival in patients with hepatitis B-related hepatocellular carcinoma
}

\author{
CHUANG QIN, YAN GAO, JIANGFA LI, CHAO HUANG and SONGQING HE \\ Division of Hepatobiliary Surgery, The First Affiliated Hospital of Guangxi \\ Medical University, Nanning, Guangxi 530021, P.R. China
}

Received July 3, 2020; Accepted March 19, 2021

DOI: $10.3892 / \mathrm{ol} .2021 .12748$

\begin{abstract}
The association between the serum levels of cancer antigen 125 (CA125; also termed MUC16) and the prognosis of patients with hepatocellular carcinoma (HCC) has not been widely reported to date. The aim of the present study was to determine the association between preoperative serum CA125 levels and prognosis of patients with hepatitis B virus (HBV)-related HCC after hepatectomy. The study included 306 patients with HBV-related HCC who underwent liver resection and were classified into four subgroups based on their baseline CA125 and $\alpha$-fetoprotein (AFP) levels. The perioperative clinical data were compared and analyzed. Kaplan-Meier and Cox regression analyses were performed to determine the associations between patient clinicopathological characteristics and survival. The results revealed that the median follow-up time was 35 months. Patients with low preoperative serum CA125 levels presented with improved 3-year disease-free survival (DFS) (79.3 vs. 75.7\%; $\mathrm{P}=0.278$ ) and overall survival (OS) $(84.4$ vs. $77.1 \% ; \mathrm{P}=0.001)$ rates compared with those among patients with high preoperative serum CA125 levels. High preoperative serum CA125 levels were a risk factor associated with short DFS and OS rates in all patients. In patients with baseline AFP levels $>100$ $\mathrm{ng} / \mathrm{ml}$, low preoperative serum CA125 levels were significantly associated with prolonged DFS and OS rates (log-rank test $\mathrm{P}=0.002$ and $\mathrm{P}=0.005$, respectively). In patients with AFP
\end{abstract}

Correspondence to: Dr Songqing He, Division of Hepatobiliary Surgery, The First Affiliated Hospital of Guangxi Medical University, 6 Shuangyong Road, Nanning, Guangxi 530021, P.R. China

E-mail: dr_hesongqing@163.com

Abbreviations: $\mathrm{HBV}$, hepatitis B virus; HCC, hepatocellular carcinoma; AST, aspartate aminotransferase; ALT, alanine aminotransferase; AFP, $\alpha$-fetoprotein

Key words: HBV-related HCC, hepatectomy, cancer antigen 125, $\alpha$-fetoprotein levels $\leq 100 \mathrm{ng} / \mathrm{ml}$, no significant differences were observed in DFS or OS rates between the high and low preoperative serum CA125 groups. Patients with high preoperative serum CA125 and AFP levels exhibited the worst prognosis (low DFS and OS rates). In conclusion, high baseline CA125 levels may be associated with a poor prognosis in patients with HBV-related HCC.

\section{Introduction}

Hepatocellular carcinoma (HCC) is one of the most common malignancies, and the third most common cause of cancerassociated mortality worldwide (1). In 2018, the estimated global incidence rate of liver cancer per 100,000 person-years was 9.3, and the corresponding mortality rate was 8.5 ; HCC accounts for $75-80 \%$ of liver cancer cases (2). In addition, chronic hepatitis B virus (HBV) infections account for $75-80 \%$ of HCC cases worldwide (3); in China, HBV infections account for $>80 \%$ of all HCC cases (4). Radical resection of liver cancer and liver transplantation are the current potential curative treatments for selected patients with HCC based on the Milan Criteria $(5,6)$. However, long-term surgical outcomes remain unsatisfactory due to high tumor recurrence rates $(7,8)$. Previous studies have reported that liver- and tumor-related characteristics such as cirrhosis, tumor size, number and vascular invasion are definitive risk factors associated with long-term survival after curative resection of $\operatorname{HCC}(9,10)$.

Cancer antigen 125 (CA125) is a high-molecular-weight glycoprotein identified with a murine monoclonal antibody against epithelial ovarian cancer (11), and serum CA125 levels have been proposed as a marker for monitoring the course of disease in patients with epithelial ovarian cancer (12). In addition, CA125 is used for the diagnosis and prognosis of various types of tumor, such as ovarian cancer, cholangiocarcinoma and lung cancer (13-16). Previous studies have demonstrated that CA125 levels are high in patients with acute and chronic liver disease, particularly in those with cirrhotic ascites $(17,18)$. The expression levels of serum CA125 are elevated in HCC compared with health subject and benign liver diseases, and CA125 exhibits $92 \%$ sensitivity for the diagnosis of HCC; however, the specificity is only $48.5 \%$, which is significantly lower compared with that of $\alpha$-fetoprotein (AFP) (19). Despite 
the numerous biomarkers proposed for HCC, such as AFP-L3, des- $\gamma$-carboxy prothrombin and osteopontin (20), the first one that was identified, AFP, remains the most utilized. Previous studies have demonstrated that CA125 provides a reference value for the prognostic evaluation of patients with various types of tumor, such as pancreatic, epithelial ovarian and lung cancer (21-23). Zhou et al (24) have reported that high preoperative serum CA125 levels predict a large tumor diameter and poor prognosis following liver resection in patients with HCC with AFP levels $\leq 200 \mathrm{ng} / \mathrm{ml}$. To date, the association between CA125 levels and prognosis of patients with HBV-related HCC has not been reported in detail; although certain retrospective reports have identified an association between them $(13,24)$, further studies are needed to confirm this. Based on the worldwide predominance of $\mathrm{HBV}$-related HCC, it is essential to investigate the association between $\mathrm{CA} 125$ and prognosis in HBV-related HCC.

To address this question, the present study retrospectively analyzed the records of 306 patients with HBV-related HCC treated by curative resection and assessed the potential associations between CA125 levels prior to tumor resection and post-operative disease-free survival (DFS) and overall survival (OS).

\section{Materials and methods}

Patients. Between April 2013 and October 2018, a retrospective study was conducted using the data from 466 consecutive patients (392 men, 74 women; median age, 54.4 years) with HBV-related HCC who underwent hepatic resection at Liuzhou People's Hospital Affiliated to Guangxi Medical University (Liuzhou, China). The inclusion criteria were as follows: i) Patients $\geq 18$ years; ii) HCC was confirmed by postoperative pathologic examination; iii) hepatitis B surface antigen was positive; iv) patient agreed to liver resection; v) curative treatment (pathological confirmation of negative resection margin); and vi) no local recurrence within 2 months after surgery. The exclusion criteria were as follows: i) Metastatic or recurrent liver cancer was confirmed by postoperative pathological examination; ii) hepatitis $\mathrm{C}$ virus or human immunodeficiency virus; iii) significant lesions of the heart, lung or kidney; iv) patient received transarterial chemoembolization or other antitumor therapy before surgery; and v) severe complications or adverse events (including postoperative mortality) within 2 months following surgery. Based on these criteria, 160 patients were excluded, and the remaining 306 patients were enrolled. The patient selection flow diagram is presented in Fig. 1. The Clinical Research Ethics Committee of Liuzhou People's Hospital Affiliated to Guangxi Medical University approved the study, and written informed consent was obtained from each patient before surgery.

All patients received conventional perioperative prophylactic antibiotics, drugs inhibiting gastric acid secretion, liver protection therapy and nutritional support. The primary outcome of the study was DFS and OS after hepatectomy. Secondary outcomes included tumor characteristics, intraoperative blood loss and intraoperative transfusion.

Based on a previous study, an AFP level of $100 \mathrm{ng} / \mathrm{ml}$ was selected as the stratification cutoff point (25). According to the stratification of preoperative serum CA125 and AFP levels, the patients were classified into four subgroups: i) Subgroup 1, CA125 $\leq 15 \mathrm{U} / \mathrm{ml}$ and AFP $\leq 100 \mathrm{ng} / \mathrm{ml}(\mathrm{n}=95)$; ii) subgroup 2, CA $125 \leq 15 \mathrm{U} / \mathrm{ml}$ and AFP $>100 \mathrm{ng} / \mathrm{ml}(\mathrm{n}=75)$; iii) subgroup $3, \mathrm{CA} 125>15 \mathrm{U} / \mathrm{ml}$ and $\mathrm{AFP} \leq 100 \mathrm{ng} / \mathrm{ml}(\mathrm{n}=57)$; and iv) subgroup 4, CA125 >15 U/ml and AFP >100 ng/ml $(n=79)$. Patients received antiviral therapy conforming to the Chronic Hepatitis B Practice Guidelines of the Asian Pacific Association for the Study of the Liver (26). Antiviral therapy was administered before or after surgery (oral $100 \mathrm{mg}$ lamivudine, $10 \mathrm{mg}$ adefovir dipivoxil or $0.5 \mathrm{mg}$ entecavir daily).

Clinical diagnosis and definitions. Diagnosis of HCC was confirmed by pathology. OS was calculated from the day of surgery until the day of mortality or last contact. The DFS was defined as the time from the day of surgery to the day of confirmed tumor recurrence, or from the day of surgery to the day of mortality or last contact for patients who did not experience recurrent disease.

Biochemical tests and follow-up. Patient blood tests were performed in the morning of the second day after hospital admission. Baseline examinations (within 7 days prior to surgery) included regular routine blood, liver function, blood coagulation function, hepatitis B surface antigen (HBsAg), HBsAg antibody (anti-HBs), hepatitis B e antigen (HBeAg), $\mathrm{HBeAg}$ antibody (anti-HBe), antibody to hepatitis core antigen (anti-HBc), AFP and CA125 concentration, and HBV DNA level tests. Serum HBV viral loads were measured using a PCR HBV monitoring kit (Roche Diagnostics K.K) with a lower detection limit of $200 \mathrm{IU} / \mathrm{ml}$. The concentration of serum CA125 and AFP was detected by ELISA (cat. nos. Q8WX17 and E-EL-H0070c; Wuhan Huamei Biotech Co., Ltd) according to the manufacturer's instructions.

Patients were followed up at Liuzhou People's Hospital every 3 months during the first postoperative year and every 3-6 months thereafter. The follow-up period ended on August 12, 2019. Blood tests and liver ultrasonography were performed during each visit by independent doctors. A computed tomography (CT) scan of the abdomen was performed every 6 months. If recurrence was suspected, CT or magnetic resonance imaging was immediately performed to confirm the diagnosis. Patients with confirmed recurrence were subjected to further treatment. If the recurrent tumor was localized, a second liver resection, radiofrequency ablation or percutaneous ethanol injection was suggested. If the recurrent tumor was multiple or diffuse, transcatheter arterial chemoembolization (TACE) was performed. Treatment decisions were based on the pattern of recurrence and liver function reserve.

Statistical analysis. For the demographic data, normally distributed continuous data are presented as the mean $\pm \mathrm{SD}$, and non-normally distributed continuous data are presented as the median and interquartile range. Categorical variables are presented as percentages. Statistical analyses were conducted using the independent samples Student's t-test (or Mann-Whitney U test for non-normally distributed data), one-way analysis of variance (or Kruskal-Wallis test for non-normally distributed data) and the $\chi^{2}$ test, as appropriate. 


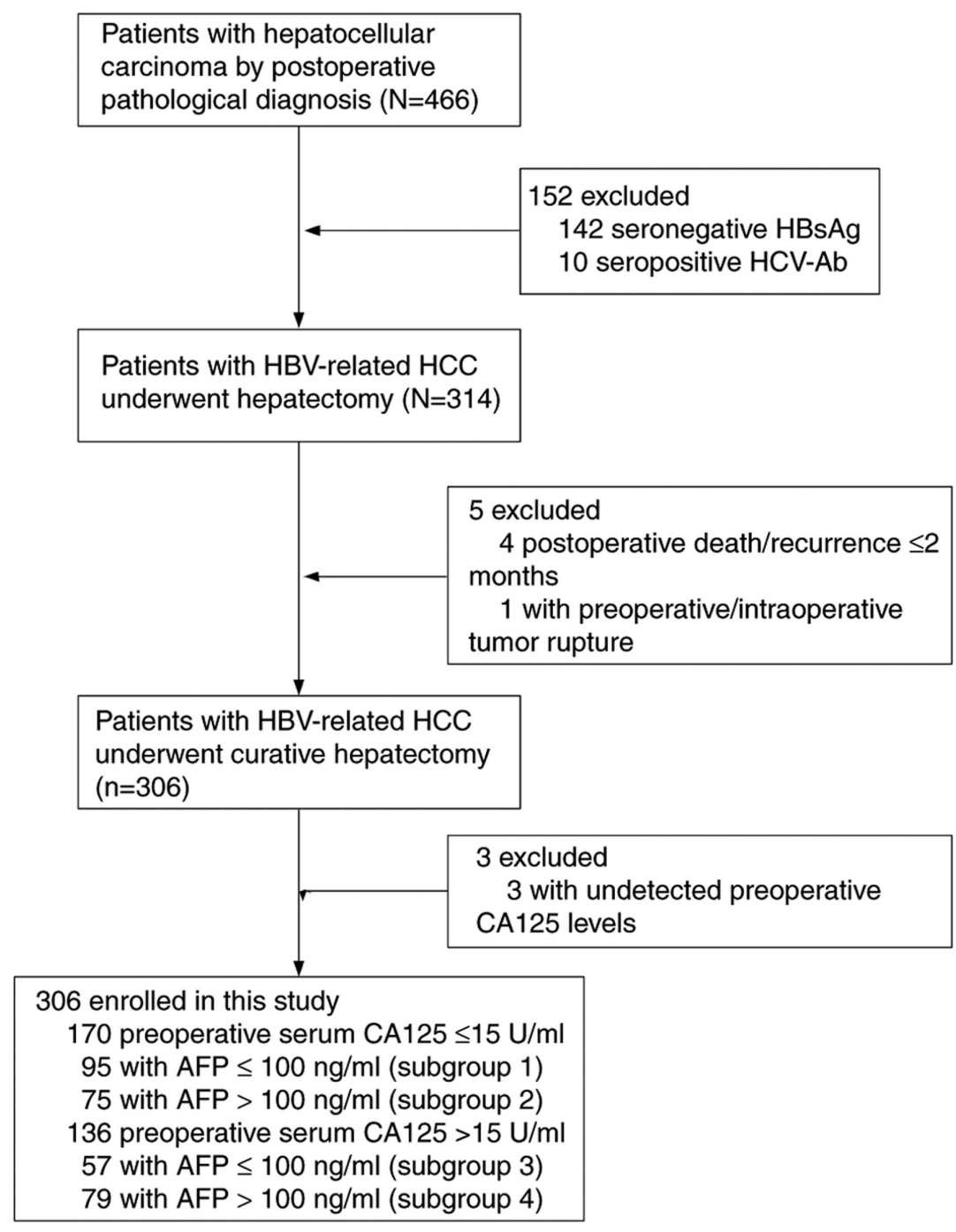

Figure 1. Selection of patients with HBV-related HCC undergoing curative resection. HCC, hepatocellular carcinoma; CA125, cancer antigen 125; HBsAg, hepatitis B surface antigen; HCV-Ab, hepatitis C virus antibody; HBV, hepatitis B virus; AFP, $\alpha$-fetoprotein.

DFS and OS were assessed using Kaplan-Meier analysis, and log-rank tests were used to evaluate differences between groups. The Bonferroni post hoc test was used for multiple comparisons. For the laboratory parameters, the cutoff values were the upper limit of the normal values. Univariate and multivariate analyses were performed by the Cox proportional hazards regression model. Following the univariate analysis, multivariate analysis of survival was performed. All analyses were conducted with SPSS 23.0 software for windows (IBM Corp.). $\mathrm{P}<0.05$ was considered to indicate a statistically significant difference.

\section{Results}

Basis patient characteristics. The median follow-up time of the patients included in the present study was 35.0 months. Table I summarizes the characteristics of the patients in the high $(>15 \mathrm{U} / \mathrm{ml})$ and low $(\leq 15 \mathrm{U} / \mathrm{ml})$ preoperative serum CA125 groups. The majority of the assessed variables were similar between the two groups. However, significant differences were noted in the platelet count, bilirubin, AFP and serum total bilirubin levels, which may be due to the patients with high AFP levels or severe liver damage having a tendency for long-term chronic liver disease and high serum CA125 levels. As presented in Table II, patients with high preoperative serum AFP levels were younger compared with those with low preoperative serum AFP levels (subgroup 1 vs. $2, \mathrm{P}=0.001$; subgroup 3 vs. $4, \mathrm{P}<0.001$ ). Patients with the same baseline AFP levels but with high serum CA125 levels presented with poorer liver function compared with that in patients with low serum CA125 levels. There were no significant differences in the preoperative liver function between the preoperative high and low serum AFP level groups when the baseline CA125 levels were the same.

\section{DFS and OS analysis}

DFS. Patients in the low preoperative CA125 group presented with higher DFS rates compared with those in the high preoperative CA125 group ( $\mathrm{P}=0.002$; Fig. 2). The 1-, 2- and 3 -year DFS rates in the low preoperative CA125 group were $93.5,87.0$ and $79.3 \%$, respectively, whereas the corresponding rates in the high preoperative CA125 group were 100.0, 84.6 and $75.7 \%$, respectively. Univariate analysis results demonstrated that male sex, platelet count $<100 \times 10^{9} / 1$, TNM 
Table I. Comparison of clinicopathological characteristics between two groups of patients stratified by the CA125 levels.

\begin{tabular}{|c|c|c|c|c|}
\hline Variable & Total, $n=306$ & $\mathrm{CA} 125>15 \mathrm{U} / \mathrm{ml}, \mathrm{n}=136$ & $\mathrm{CA} 125 \leq 15 \mathrm{U} / \mathrm{ml}, \mathrm{n}=170$ & P-value \\
\hline Age, years, mean \pm SD & $51.9 \pm 11.7$ & $50.7 \pm 12.1$ & $52.8 \pm 11.4$ & $0.116^{\mathrm{a}}$ \\
\hline Sex, male, $\mathrm{n}(\%)$ & $261(85.3 \%)$ & $114(83.8 \%)$ & $147(86.5 \%)$ & 0.516 \\
\hline Diabetes, n (\%) & $68(22.2 \%)$ & $37(27.2 \%)$ & $31(18.2 \%)$ & 0.061 \\
\hline Hypertension, n (\%) & $45(14.7 \%)$ & $22(16.2 \%)$ & $23(13.5 \%)$ & 0.516 \\
\hline Platelet count, $\mathrm{x} 10^{9} / 1$ & $178(135-235)$ & 199 (149-257) & $171(129-216)$ & $0.004^{\mathrm{b}}$ \\
\hline $\mathrm{TBIL}, \mu \mathrm{mol} / \mathrm{l}$ & $12.7(9.8-18.0)$ & $13.7(10.6-20.9)$ & $11.8(9.2-16.0)$ & $0.001^{\mathrm{b}}$ \\
\hline ALT, IU/1 & $54(30-95)$ & $56(33-94)$ & $45(27-96)$ & $0.440^{\mathrm{b}}$ \\
\hline AST, IU/1 & $80(37-130)$ & $82(42-132)$ & $72(32-125)$ & $0.503^{\mathrm{b}}$ \\
\hline $\mathrm{ALB}, \mathrm{g} / \mathrm{l}$, mean $\pm \mathrm{SD}$ & $37.9 \pm 4.47$ & $36.8 \pm 4.38$ & $38.8 \pm 4.33$ & $<0.001^{\mathrm{a}}$ \\
\hline $\mathrm{PT}, \mathrm{sec}$, mean $\pm \mathrm{SD}$ & $14.9 \pm 1.67$ & $15.0 \pm 1.69$ & $14.9 \pm 1.66$ & $0.507^{\mathrm{a}}$ \\
\hline HBV DNA, IU/ml (\%) & & & & 0.085 \\
\hline$\leq 2,000$ & $193(63.1 \%)$ & $93(68.4 \%)$ & $100(58.8 \%)$ & \\
\hline$>2,000$ & $113(36.9 \%)$ & $43(31.6 \%)$ & $70(41.2 \%)$ & \\
\hline AFP, ng/ml & & & & 0.015 \\
\hline$\leq 100$ & $152(49.7 \%)$ & $57(41.9 \%)$ & $95(55.9 \%)$ & \\
\hline$>100$ & $154(50.3 \%)$ & $79(58.1 \%)$ & $75(44.1 \%)$ & \\
\hline ASA & & & & 0.286 \\
\hline I & $189(61.8 \%)$ & $82(60.3 \%)$ & $107(62.9 \%)$ & \\
\hline II & $105(34.3 \%)$ & $46(33.8 \%)$ & $59(56.2 \%)$ & \\
\hline III & $12(3.9 \%)$ & $8(5.9 \%)$ & $4(2.4 \%)$ & \\
\hline Child-Pugh grade & & & & 0.088 \\
\hline A & $243(79.4 \%)$ & $114(83.8 \%)$ & $129(75.9 \%)$ & \\
\hline B & $63(20.6 \%)$ & $22(16.2 \%)$ & $41(24.1 \%)$ & \\
\hline Cirrhosis, n (\%) & $259(84.6 \%)$ & $113(83.1 \%)$ & $146(85.9 \%)$ & 0.501 \\
\hline Ascites, n (\%) & $80(26.1 \%)$ & $41(31.1 \%)$ & $39(22.9 \%)$ & 0.154 \\
\hline Tumor diameter, $\mathrm{cm}$ & $5.5(3.7-9.0)$ & $7.0(4.4-11.1)$ & $5.0(3.5-7.5)$ & $<0.001$ \\
\hline Tumor number, n (\%) & & & & 0.186 \\
\hline Solitary & $238(77.8 \%)$ & $101(74.3 \%)$ & $137(80.6 \%)$ & \\
\hline Multiple & $68(22.2 \%)$ & $35(25.7 \%)$ & $33(19.4 \%)$ & \\
\hline TNM stage, n (\%) & & & & $<0.001$ \\
\hline I & $152(49.7 \%)$ & $55(40.4 \%)$ & $97(57.1 \%)$ & \\
\hline II & $50(16.3 \%)$ & $18(13.2 \%)$ & $32(18.8 \%)$ & \\
\hline III & $104(34.0 \%)$ & $63(46.3 \%)$ & $41(24.1 \%)$ & \\
\hline Venous invasion $(\mathrm{n}, \%)$ & $114(36.3 \%)$ & $80(37.6 \%)$ & $34(33.7 \%)$ & 0.503 \\
\hline Tumor differentiation $(\mathrm{n}, \%)$ & & & & 0.352 \\
\hline Well differentiated & $31(10.1 \%)$ & $10(7.4 \%)$ & $21(12.4 \%)$ & \\
\hline Moderately differentiated & $258(84.3 \%)$ & $118(86.8 \%)$ & $140(82.4 \%)$ & \\
\hline Poorly differentiated & $17(5.6 \%)$ & $8(5.9 \%)$ & $9(5.3 \%)$ & \\
\hline Intraoperative blood loss, ml & $500(200-825)$ & $500(200-1,000)$ & $450(200-800)$ & $0.058^{\mathrm{b}}$ \\
\hline Intraoperative transfusion $(\mathrm{n}, \%)$ & $118(38.6 \%)$ & $65(47.8 \%)$ & $53(31.2 \%)$ & 0.003 \\
\hline Surgery time, $\min$ & $180(144-210)$ & $180(150-235)$ & $180(140-206)$ & $0.162^{b}$ \\
\hline TACE, n (\%) & & & & 0.175 \\
\hline$\leq 3$ & $268(87.6 \%)$ & $123(90.4 \%)$ & $145(85.3 \%)$ & \\
\hline$>3$ & $38(12.4 \%)$ & $13(9.6 \%)$ & $25(14.7 \%)$ & \\
\hline
\end{tabular}

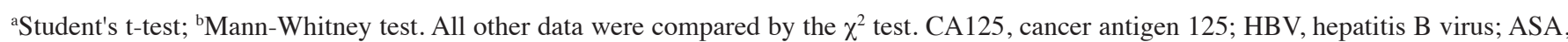
American Society of Anesthesiologists; TNM, Tumor-Node-Metastasis; ALT, alanine aminotransferase; AST, aspartate aminotransferase; PT, prothrombin time; TBIL, serum total bilirubin; AFP, $\alpha$-fetoprotein; TACE, transcatheter arterial chemoembolization; ALB, serum albumin .

stage III, tumor diameter $>5 \mathrm{~cm}$, multiple tumors, venous invasion, tumor differentiation, intraoperative blood loss $>1,000 \mathrm{ml}$, intraoperative transfusion and high serum CA125 levels were independent risk factors associated with DFS
(Table III). Multivariate analysis revealed that tumor diameter $>5 \mathrm{~cm}$, multiple tumors and vascular invasion at the time of resection were independent risk factors associated with short DFS (Table III). 
Table II. Comparison of clinicopathologic characteristics among the patient subgroups stratified by CA125 and AFP levels.

\begin{tabular}{|c|c|c|c|c|c|c|c|c|}
\hline Variables & $\begin{array}{l}\text { Subgroup } 1, \\
n=95\end{array}$ & $\begin{array}{l}\text { Subgroup } 2, \\
\quad n=75\end{array}$ & $\begin{array}{c}\text { Subgroup } 3, \\
n=57\end{array}$ & $\begin{array}{c}\text { Subgroup } 4, \\
n=79\end{array}$ & $\mathrm{P}_{1}$ & $\mathrm{P}_{2}$ & $\mathrm{P}_{3}$ & $\mathrm{P}_{4}$ \\
\hline Age, years, mean \pm SD & $55.3 \pm 10.2$ & $49.7 \pm 12.0$ & $56.4 \pm 11.7$ & $46.6 \pm 10.8$ & $0.001^{\mathrm{a}}$ & $0.577^{\mathrm{a}}$ & $0.104^{\mathrm{a}}$ & $<0.001^{\mathrm{a}}$ \\
\hline Sex, male, $\mathrm{n}(\%)$ & $79(83.2 \%)$ & $68(90.7 \%)$ & $51(89.5 \%)$ & $63(79.7 \%)$ & 0.155 & 0.284 & 0.057 & 0.129 \\
\hline Hypertension, n (\%) & $16(16.8 \%)$ & $4(5.3 \%)$ & $7(12.3 \%)$ & $5(6.3 \%)$ & 0.021 & 0.447 & - & 0.227 \\
\hline Diabetes, n (\%) & $19(10.0 \%)$ & $12(16.0 \%)$ & $16(28.1 \%)$ & $21(26.6 \%)$ & 0.502 & 0.253 & 0.110 & 0.847 \\
\hline Platelet count, $\times 10^{9} / 1$ & $177.6 \pm 62.2$ & $178.7 \pm 66.8$ & $178.0 \pm 74.2$ & $221.0 \pm 93.8$ & $0.912^{\mathrm{a}}$ & $0.970^{\mathrm{a}}$ & $0.002^{\mathrm{a}}$ & $0.005^{\mathrm{a}}$ \\
\hline $\mathrm{TBIL}, \mu \mathrm{mol} / \mathrm{l}$ & $11.6(9.4-15.4)$ & $11.9(8.8-16.6)$ & $16.1(11.6-21.8)$ & $12.8(9.9-20.8)$ & $0.787^{\mathrm{b}}$ & $0.001^{\mathrm{b}}$ & $0.097^{b}$ & $0.083^{\mathrm{b}}$ \\
\hline ALT, IU/1 & $40(25-84)$ & $66(36-114)$ & $49(29-94)$ & $58(37-85)$ & $0.008^{\mathrm{b}}$ & $0.245^{\mathrm{b}}$ & $0.548^{b}$ & $0.457^{\mathrm{b}}$ \\
\hline AST, IU/1 & $71(31-145)$ & $87(43-126)$ & $80(41-119)$ & $84(48-132)$ & $0.350^{\mathrm{b}}$ & $0.812^{\mathrm{b}}$ & $0.622^{b}$ & $0.260^{\mathrm{b}}$ \\
\hline PT, sec & $14.8 \pm 1.6$ & $15 \pm 1.6$ & $15.3 \pm 1.7$ & $14.8 \pm 1.6$ & $0.520^{\mathrm{a}}$ & $0.086^{\mathrm{a}}$ & $0.510^{\mathrm{a}}$ & $0.088^{\mathrm{a}}$ \\
\hline HBV DNA IU/ml , n (\%) & & & & & 0.971 & 0.085 & 0.078 & 0.266 \\
\hline$\leq 2,000$ & $39(41.1 \%)$ & $31(41.3 \%)$ & $21(36.8 \%)$ & $22(27.8 \%)$ & & & & \\
\hline$>2,000$ & $56(58.9 \%)$ & $44(58.7 \%)$ & $36(63.2 \%)$ & $57(72.2 \%)$ & & & & \\
\hline Albumin, g/l & $39.3(36.6-40.7)$ & $38.8(36.5-42.0)$ & $36.4(33.5-39.3)$ & $37.5(33.3-40.4)$ & $0.651^{\mathrm{b}}<$ & $<0.001^{\mathrm{b}}$ & $0.006^{\mathrm{b}}$ & $0.225^{\mathrm{b}}$ \\
\hline ASA, n (\%) & & & & & 0.202 & 0.326 & 0.213 & 0.231 \\
\hline I & $60(63.2 \%)$ & $47(62.7 \%)$ & $30(52.6 \%)$ & $52(65.8 \%)$ & & & & \\
\hline II & $31(32.6 \%)$ & $28(37.3 \%)$ & $22(38.6 \%)$ & $24(30.4 \%)$ & & & & \\
\hline III & $4(4.2 \%)$ & 0 & $5(8.8 \%)$ & $3(3.8 \%)$ & & & & \\
\hline Child-Pugh grade, n (\%) & & & & & 0.293 & 0.424 & 0.084 & 0.917 \\
\hline A & $75(78.9 \%)$ & $54(72.0 \%)$ & $48(84.2 \%)$ & $66(83.5 \%)$ & & & & \\
\hline $\mathrm{B}$ & $20(21.1 \%)$ & $21(28.0 \%)$ & $9(15.8 \%)$ & $13(16.5 \%)$ & & & & \\
\hline Cirrhosis & $78(82.1 \%)$ & $68(90.7 \%)$ & $48(84.2 \%)$ & $65(82.3 \%)$ & 0.111 & 0.739 & 0.129 & 0.767 \\
\hline Ascites & $23(24.2 \%)$ & $16(21.3 \%)$ & $17(29.8 \%)$ & $24(30.4 \%)$ & 0.658 & 0.447 & 0.201 & 0.944 \\
\hline TNM stage, n (\%) & & & & & 0.027 & 0.162 & 0.007 & 0.005 \\
\hline I & $57(60.0 \%)$ & $40(53.3 \%)$ & $30(52.7 \%)$ & $25(31.6 \%)$ & & & & \\
\hline II & $22(23.2 \%)$ & $10(13.3 \%)$ & $10(17.5 \%)$ & $8(10.2 \%)$ & & & & \\
\hline III & $16(16.8 \%)$ & $25(33.3 \%)$ & $17(29.8 \%)$ & $46(58.2 \%)$ & & & & \\
\hline Tumor diameter, $\mathrm{cm}$ & $5.5 \pm 3.4$ & $6.2 \pm 3.8$ & $6.2 \pm 4.0$ & $8.9 \pm 4.4$ & 0.247 & 0.302 & $<0.001$ & $<0.001$ \\
\hline Tumor number, n (\%) & & & & & 0.010 & 0.464 & 0.004 & 0.289 \\
\hline Solitary & $70(73.7 \%)$ & $67(89.3 \%)$ & $45(78.9 \%)$ & $56(70.9 \%)$ & & & & \\
\hline Multiple & $25(26.3 \%)$ & $8(10.7 \%)$ & $12(21.1 \%)$ & $23(29.1 \%)$ & & & & \\
\hline Venous invasion, $\mathrm{n}(\%)$ & $27(28.4 \%)$ & $28(37.3 \%)$ & $18(31.6 \%)$ & $39(49.4 \%)$ & 0.217 & 0.680 & 0.132 & 0.038 \\
\hline Tumor differentiation, $\mathrm{n}(\%)$ & & & & & 0.083 & 0.945 & 0.185 & 0.004 \\
\hline Well differentiated & $16(16.8 \%)$ & $5(6.7 \%)$ & $9(15.8 \%)$ & $1(1.3 \%)$ & & & & \\
\hline Moderately differentiated & $73(76.8 \%)$ & $67(89.3 \%)$ & $45(78.9 \%)$ & $73(92.4 \%)$ & & & & \\
\hline Poorly differentiated & $6(6.3)$ & $3(4.0 \%)$ & $3(5.3 \%)$ & $5(6.3)$ & & & & \\
\hline Intraoperative blood loss & $400(200-700)$ & $400(200-900)$ & $400(200-1,000)$ & $600(300-1,200)$ & $0.308^{\mathrm{b}}$ & $0.906^{\mathrm{b}}$ & $0.045^{\mathrm{b}}$ & $0.020^{\mathrm{b}}$ \\
\hline Intraoperative & $24(25.3 \%)$ & $29(38.7 \%)$ & $22(38.6 \%)$ & $43(54.4 \%)$ & 0.061 & 0.083 & 0.050 & 0.068 \\
\hline $\begin{array}{l}\text { Surgery time } \\
\text { transfusion, n (\%) }\end{array}$ & $180(135-210)$ & $180(140-202)$ & $180(125-194)$ & $180(150-240)$ & $0.786^{\mathrm{b}}$ & $0.825^{\mathrm{b}}$ & $0.069^{\mathrm{b}}$ & $0.034^{\mathrm{b}}$ \\
\hline TACE, n (\%) & & & & & 0.009 & 0.009 & 0.210 & 0.148 \\
\hline$\leq 3$ & $75(78.9 \%)$ & $70(93.3 \%)$ & $54(94.7 \%)$ & $69(87.3 \%)$ & & & & \\
\hline$>3$ & $20(21.1 \%)$ & $5(6.7 \%)$ & $3(5.3 \%)$ & $10(12.7 \%)$ & & & & \\
\hline
\end{tabular}

aStudent's t-test; 'Mann-Whitney test. All other data were compared by the $\chi^{2}$ test. Subgroup 1, preoperative serum CA125 $\leq 15 \mathrm{U} / \mathrm{ml}$ and preoperative serum AFP $\leq 100 \mathrm{ng} / \mathrm{ml}$; subgroup 2, preoperative serum CA125 $\leq 15 \mathrm{U} / \mathrm{ml}$ and preoperative serum AFP $>100 \mathrm{ng} / \mathrm{ml}$; subgroup 3 , preoperative serum CA125 >15 U/ml and preoperative serum AFP $\leq 100 \mathrm{ng} / \mathrm{ml}$; subgroup 4, preoperative serum CA125 >15 U/ml and preoperative serum AFP $>100 \mathrm{ng} / \mathrm{ml}$. P1, P-value of subgroup 1 vs. 2; P2, P-value of subgroup 1 vs. 3; P3, P-value of subgroup 2 vs. 4; P4, P-value of subgroup 3 vs. 4. CA125, cancer antigen 125; HBV, hepatitis B virus; ASA, American Society of Anesthesiologists; TNM, Tumor-Node-Metastasis; ALT, alanine aminotransferase; AST, aspartate aminotransferase; PT, prothrombin time; TBIL, serum total bilirubin; AFP, $\alpha$-fetoprotein; TACE, transcatheter arterial chemoembolization; ALB, serum albumin. 


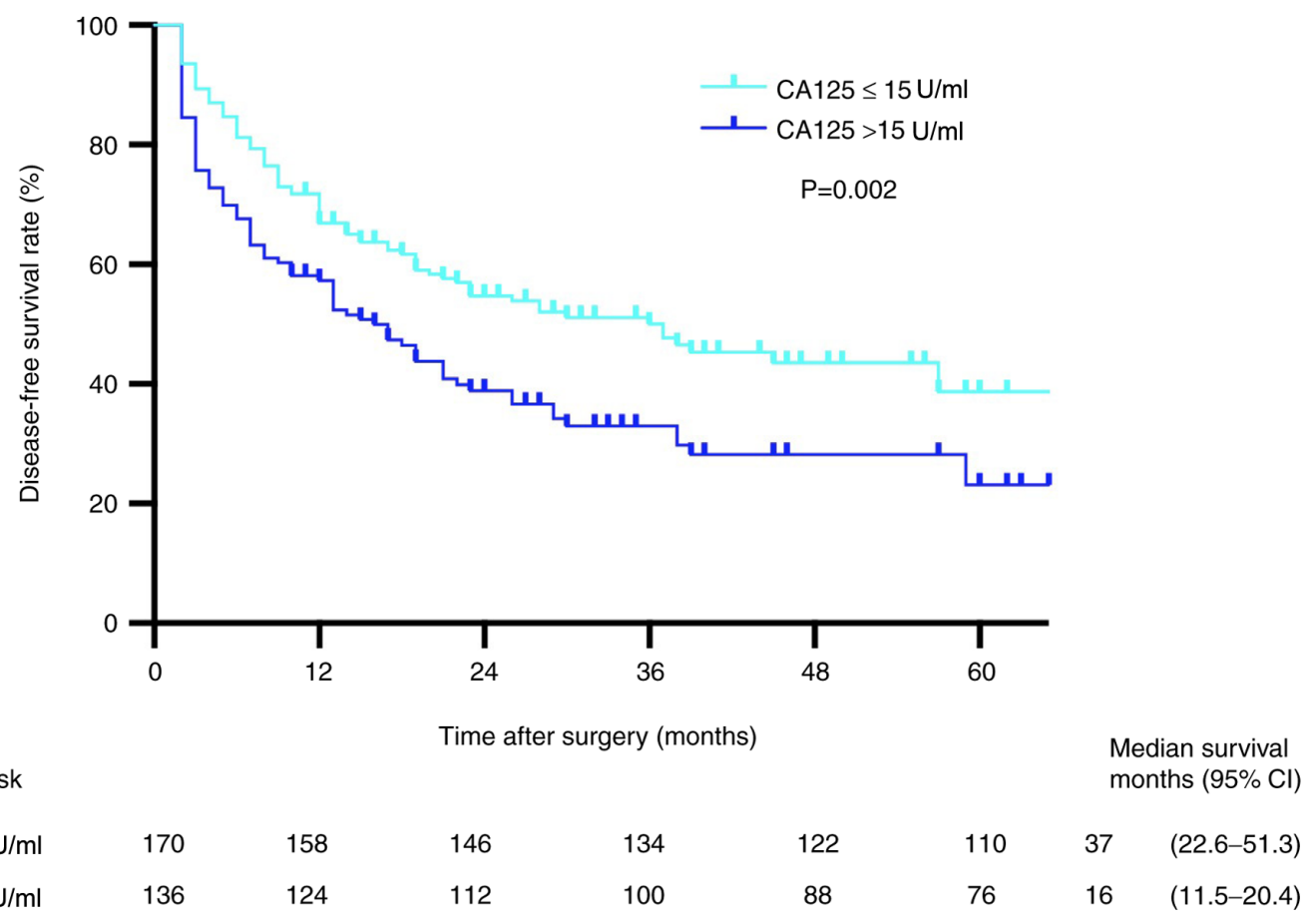

Figure 2. Kaplan-Meier curves for disease-free survival in patients with hepatitis B virus-related hepatocellular carcinoma (n=306). CA125, cancer antigen 125; $\mathrm{CI}$, confidence interval.

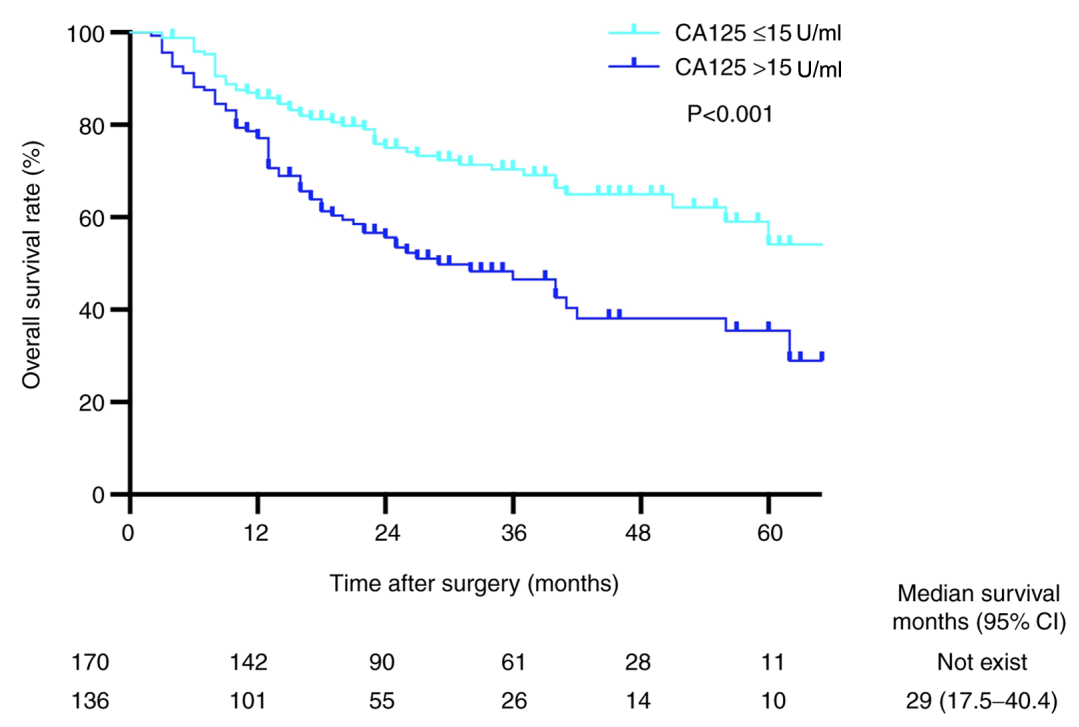

Figure 3. Kaplan-Meier curves for overall survival in patients with hepatitis B virus-related hepatocellular carcinoma (n=306). CA125, cancer antigen 125; $\mathrm{CI}$, confidence interval.

$O S$. Kaplan-Meier analysis revealed that the OS rates in patients with preoperative serum CA125 levels $\leq 15 \mathrm{U} / \mathrm{ml}$ were significantly higher compared with those in patients with preoperative serum CA125 levels $>15 \mathrm{U} / \mathrm{ml}$ ( $\mathrm{P}<0.001$; Fig. 3 ). The 1-, 2- and 3-year OS rates in the low preoperative serum CA125 group were 95.2, 86.9 and $84.4 \%$, respectively, whereas the corresponding rates in the high preoperative serum CA125 group were $91.2,83.1$ and $77.1 \%$, respectively. Univariate analysis revealed that sex, AFP $>100 \mathrm{ng} / \mathrm{ml}$, TNM stage III, tumor diameter $>5 \mathrm{~cm}$, multiple tumors, venous invasion, tumor differentiation, intraoperative blood loss $>1,000 \mathrm{ml}$, intraoperative transfusion and serum CA125 levels were independent risk factors associated with OS (Table III). The results of the multivariate analysis demonstrated that tumor diameter $>5 \mathrm{~cm}$, multiple tumors, venous invasion and preoperative serum CA125 levels were independent risk factors associated with short OS (Table III).

Analysis based on the stratification of preoperative serum CA125 and AFP levels. All patients were stratified by the baseline AFP and preoperative serum CA125 levels, and the association of CA125 levels with long-term prognosis was evaluated in each stratum. Table II summarizes the characteristics of these four subgroups. 
Table III. Univariate and multivariate analyses of DFS and OS in all patients $(n=306)$.

\begin{tabular}{|c|c|c|c|c|c|c|c|c|}
\hline \multirow[b]{3}{*}{ Index } & \multicolumn{4}{|c|}{ DFS } & \multicolumn{4}{|c|}{ OS } \\
\hline & \multicolumn{2}{|c|}{$\begin{array}{l}\text { Univariate } \\
\text { analysis }\end{array}$} & \multicolumn{2}{|c|}{ Multivariate analysis } & \multicolumn{2}{|c|}{$\begin{array}{l}\text { Univariate } \\
\text { analysis }\end{array}$} & \multicolumn{2}{|c|}{ Multivariate analysis } \\
\hline & HR & P-value & $\mathrm{HR}(95 \% \mathrm{CI})$ & P-value & HR & P-value & $\mathrm{HR}(95 \% \mathrm{CI})$ & P-value \\
\hline Age & 0.763 & 0.267 & & & 0.873 & 0.623 & & \\
\hline Sex & 0.599 & 0.035 & & & 0.539 & 0.036 & & \\
\hline Hypertension & 1.325 & 0.284 & & & 1.082 & 0.795 & & \\
\hline Diabetes & 1.356 & 0.077 & & & 1.455 & 0.064 & & \\
\hline ALT & 1.274 & 0.135 & & & 0.999 & 0.439 & & \\
\hline AST & 1.076 & 0.673 & & & 1.000 & 0.822 & & \\
\hline Platelet count ( $\geq 100$ vs. $\left.<100 \times 10^{9} / 1\right)$ & 0.489 & 0.022 & & & 0.665 & 0.240 & & \\
\hline PT $(\leq 14$ vs. $>14 \mathrm{sec})$ & 1.139 & 0.430 & & & 1.047 & 0.815 & & \\
\hline TBIL ( $\leq 17$ vs. $>17 \mu \mathrm{mol} / \mathrm{l}$ ) & 1.118 & 0.504 & & & 1.193 & 0.373 & & \\
\hline HBV DNA ( $\leq 2,000$ vs. $>2,000 \mathrm{IU} / \mathrm{ml})$ & 1.325 & 0.284 & & & 1.196 & 0.356 & & \\
\hline $\operatorname{AFP}(\leq 100$ vs. $>100 \mathrm{ng} / \mathrm{ml})$ & 1.310 & 0.077 & & & 1.052 & 0.026 & & \\
\hline \multicolumn{9}{|l|}{ TNM stage } \\
\hline I & (Ref.) & (Ref.) & & & (Ref.) & (Ref.) & & \\
\hline II & 1.144 & 0.555 & & & 1.073 & 0.809 & & \\
\hline III & 1.781 & $<0.001$ & & & 2.220 & $<0.001$ & & \\
\hline \multicolumn{9}{|l|}{ ASA } \\
\hline I & (Ref.) & (Ref.) & & & (Ref.) & (Ref.) & & \\
\hline II & 1.111 & 0.515 & & & 1.115 & 0.571 & & \\
\hline III & 1.785 & 0.097 & & & 0.955 & 0.929 & & \\
\hline Child-Pugh grade (A vs. B) & 0.919 & 0.661 & & & 0.708 & 0.151 & & \\
\hline Cirrhosis & 0.959 & 0.839 & & & 1.370 & 0.254 & & \\
\hline Ascites & 1.370 & 0.058 & & & 1.192 & 0.387 & & \\
\hline Tumor diameter ( $\leq 5$ vs. $>5 \mathrm{~cm}$ ) & 2.069 & $<0.001$ & $\begin{array}{c}1.725 \\
(1.229-2.419)\end{array}$ & 0.002 & 2.377 & $<0.001$ & $\begin{array}{c}1.746 \\
(1.142-2.668)\end{array}$ & 0.010 \\
\hline Tumor number (Multiple vs. solitary) & 1.985 & $<0.001$ & $\begin{array}{c}1.751 \\
(1.253-2.446)\end{array}$ & 0.001 & 2.122 & $<0.001$ & $\begin{array}{c}1.739 \\
(1.175-2.573)\end{array}$ & 0.006 \\
\hline Venous invasion (Yes vs. no) & 1.960 & $<0.001$ & $\begin{array}{c}1.668 \\
(1.223-2.277)\end{array}$ & 0.001 & 2.280 & $<0.001$ & $\begin{array}{c}1.819 \\
(1.254-2.638)\end{array}$ & 0.002 \\
\hline \multicolumn{9}{|l|}{ Tumor differentiation } \\
\hline Well differentiated & (Ref.) & (Ref.) & & & (Ref.) & (Ref.) & & \\
\hline Moderately differentiated & 2.283 & 0.008 & & & 3.541 & 0.006 & & \\
\hline Poorly differentiated & 2.339 & 0.052 & & & 2.029 & 0.068 & & \\
\hline $\begin{array}{l}\text { Intraoperative blood loss } \\
(\leq 1,000 \text { vs. }>1,000 \mathrm{ml})\end{array}$ & 1.561 & 0.008 & & & 1.649 & 0.010 & & \\
\hline Intraoperative transfusion (Yes vs. no) & 1.453 & 0.015 & & & 1.640 & 0.007 & & \\
\hline TACE ( $\leq 3$ vs. $>3)$ & 1.102 & 0.653 & & & 0.861 & 0.578 & & \\
\hline CA125 ( $\leq 15$ vs. $>15$ U/ml) & 1.597 & 0.002 & & & 2.112 & $<0.001$ & $\begin{array}{c}1.709 \\
(1.177-2.482)\end{array}$ & 0.005 \\
\hline
\end{tabular}

DFS, disease-free survival; OS, overall survival; HBV, hepatitis B virus; ASA, American Society of Anesthesiologists; TNM, Tumor-Node-Metastasis; ALT, alanine aminotransferase; AST, aspartate aminotransferase; PT, prothrombin time; TBIL, serum total bilirubin; AFP, $\alpha$-fetoprotein; TACE, transcatheter arterial chemoembolization; CA125, cancer antigen 125; HR, hazard ratio; CI, confidence interval.

Impact of preoperative serum CA125 levels on the prognosis of patients with high baseline AFP. In the 154 patients in the high preoperative serum AFP level group (subgroups 2 and 4),
Kaplan-Meier analysis demonstrated that high preoperative serum CA125 levels were associated with lower DFS and OS rates compared with those in the low CA125 group (both 
Table IV. Univariate and multivariate analyses of DFS and OS in patients with high preoperative serum AFP $>100 \mathrm{ng} / \mathrm{ml}(\mathrm{n}=154)$.

\begin{tabular}{|c|c|c|c|c|c|c|c|c|}
\hline \multirow[b]{3}{*}{ Index } & \multicolumn{4}{|c|}{ DFS } & \multicolumn{4}{|c|}{ OS } \\
\hline & \multicolumn{2}{|c|}{$\begin{array}{l}\text { Univariate } \\
\text { analysis }\end{array}$} & \multicolumn{2}{|c|}{ Multivariate analysis } & \multicolumn{2}{|c|}{$\begin{array}{l}\text { Univariate } \\
\text { analysis }\end{array}$} & \multicolumn{2}{|c|}{ Multivariate analysis } \\
\hline & HR & P-value & $\mathrm{HR}(95 \% \mathrm{CI})$ & P-value & HR & P-value & HR $(95 \%$ CI $)$ & P-value \\
\hline Age & 0.407 & 0.126 & & & 0.419 & 0.226 & & \\
\hline Sex & 0.742 & 0.337 & & & 0.651 & 0.232 & & \\
\hline Hypertension & 1.337 & 0.528 & & & 1.447 & 0.532 & & \\
\hline Diabetes & 1.220 & 0.408 & & & 1.207 & 0.501 & & \\
\hline ALT & 1.164 & 0.511 & & & 1.516 & 0.145 & & \\
\hline AST & 1.051 & 0.838 & & & 1.351 & 0.318 & & \\
\hline Platelet count ( $\geq 100$ vs. $\left.<100 \times 10^{9} / 1\right)$ & 0.472 & 0.056 & & & 0.621 & 0.264 & & \\
\hline PT $(\leq 14$ vs. $>14 \mathrm{sec})$ & 1.075 & 0.745 & & & 1.157 & 0.573 & & \\
\hline TBIL ( $\leq 17$ vs. $>17 \mu \mathrm{mol} / \mathrm{l})$ & 1.137 & 0.578 & & & 1.148 & 0.258 & & \\
\hline HBV DNA $(\leq 2,000$ vs. $>2,000 \mathrm{IU} / \mathrm{ml})$ & 1.055 & 0.806 & & & 1.290 & 0.331 & & \\
\hline \multicolumn{9}{|l|}{ TNM stage } \\
\hline I & (Ref.) & (Ref.) & & & (Ref.) & (Ref.) & & \\
\hline II & 0.683 & 0.361 & & & 0.779 & 0.614 & & \\
\hline III & 1.961 & 0.002 & & & 1.386 & 0.001 & & \\
\hline \multicolumn{9}{|l|}{ ASA } \\
\hline I & (Ref.) & (Ref.) & & & (Ref.) & (Ref.) & & \\
\hline II & 1.109 & 0.637 & & & 0.983 & 0.946 & & \\
\hline III & 1.249 & 0.758 & & & 0.826 & 0.851 & & \\
\hline Child-Pugh grade (A vs. B) & 0.823 & 0.450 & & & 0.609 & 0.119 & & \\
\hline Cirrhosis & 1.006 & 0.984 & & & 1.397 & 0.374 & & \\
\hline Ascites & 1.391 & 0.145 & & & 1.042 & 0.882 & & \\
\hline Tumor diameter ( $\leq 5$ vs. $>5 \mathrm{~cm}$ ) & 1.779 & 0.014 & & & 2.105 & 0.008 & & \\
\hline Tumor number (Multiple vs. solitary) & 1.864 & 0.009 & & & 2.488 & 0.001 & $\begin{array}{c}1.862 \\
(1.096-3.162)\end{array}$ & 0.021 \\
\hline Venous invasion (Yes vs. no) & 2.101 & $<0.001$ & $\begin{array}{c}1.943 \\
(1.282-2.943)\end{array}$ & 0.002 & 2.104 & 0.002 & $\begin{array}{c}1.822 \\
(1.121-2.960)\end{array}$ & 0.015 \\
\hline \multicolumn{9}{|l|}{ Tumor differentiation } \\
\hline Well differentiated & (Ref.) & (Ref.) & & & (Ref.) & (Ref.) & & \\
\hline Moderately differentiated & 2.418 & 0.217 & & & 1.983 & 0.341 & & \\
\hline Poorly differentiated & 3.049 & 0.173 & & & 1.649 & 0.584 & & \\
\hline $\begin{array}{l}\text { Intraoperative blood loss } \\
(\leq 1,000 \text { vs. }>1,000 \mathrm{ml})\end{array}$ & 1.484 & 0.071 & & 1.427 & 0.155 & & & \\
\hline Intraoperative transfusion (Yes vs. no) & 1.078 & 0.717 & & & 1.218 & 0.411 & & \\
\hline TACE ( $\leq 3$ vs. $>3)$ & 0.893 & 0.735 & & & 0.840 & 0.644 & & \\
\hline CA125 ( $\leq 15$ vs. $>15 \mathrm{U} / \mathrm{ml})$ & 2.120 & $<0.001$ & $\begin{array}{c}1.965 \\
(1.286-3.004)\end{array}$ & 0.002 & 2.496 & $<0.001$ & $\begin{array}{c}2.170 \\
(1.299-3.624)\end{array}$ & 0.003 \\
\hline
\end{tabular}

DFS, disease-free survival; OS, overall survival; HBV, hepatitis B virus; ASA, American Society of Anesthesiologists; TNM, Tumor-Node-Metastasis; ALT, alanine aminotransferase; AST, aspartate aminotransferase; PT, prothrombin time; TBIL, serum total bilirubin; AFP, $\alpha$-fetoprotein; TACE, transcatheter arterial chemoembolization; CA125, cancer antigen 125; HR, hazard ratio; CI, confidence interval.

$\mathrm{P}<0.001$; Fig. 4A and B). Univariate and multivariate Cox models revealed that high preoperative serum CA125 levels along with certain tumor characteristics (TNM stage III, tumor diameter $>5 \mathrm{~cm}$, multiple tumors and the presence of venous invasion) were risk factors for short DFS and OS rates in the high preoperative serum AFP group (Table IV).
Impact of preoperative serum CA125 levels on the prognosis of patients with low baseline AFP. Kaplan-Meier analysis identified no significant differences in the OS rates between the high and low preoperative serum CA125 level groups in patients with low baseline AFP levels (subgroup 1 vs. 3, $\mathrm{P}=0.136$; Fig. 4B). Univariate analysis identified the following 
A

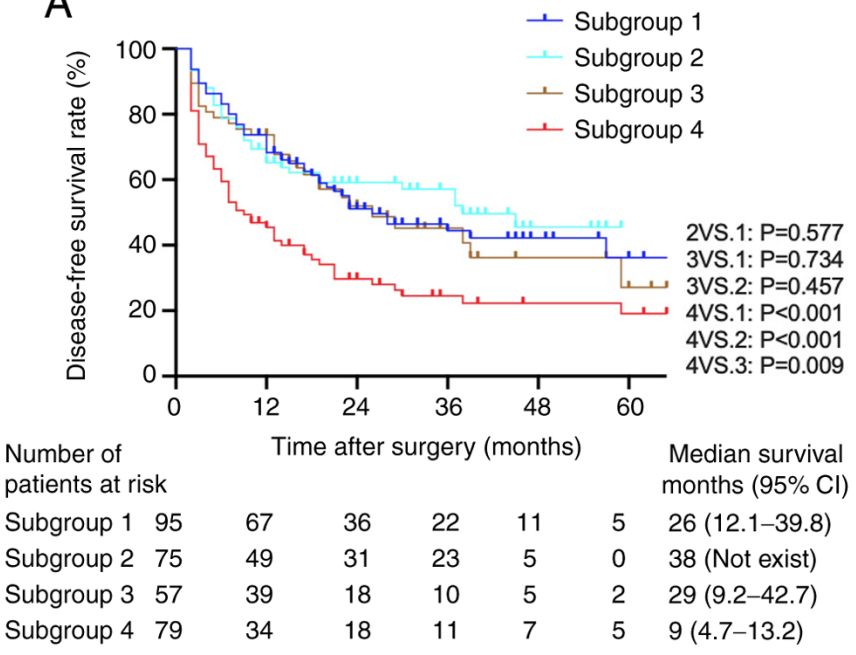

$\mathrm{B}$

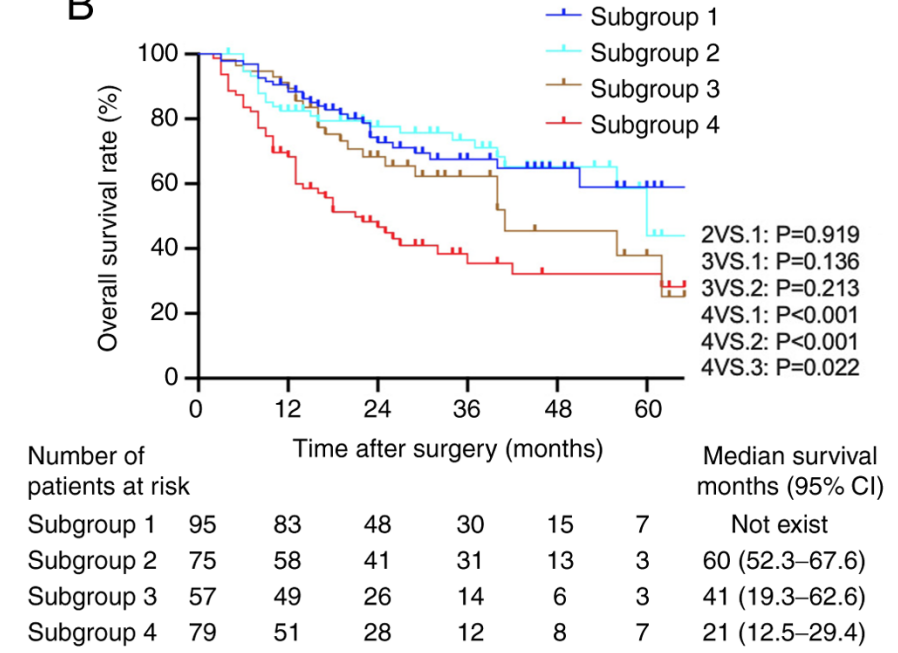

Figure 4. Kaplan-Meier survival curves of patients in high and low preoperative serum CA125 subgroups following stratification by baseline AFP levels. (A) Disease-free survival rates among the four subgroups of patients. (B) Overall survival rates among the four subgroups of patients (log-rank test: Subgroup 4 vs. $1, \mathrm{P}<0.001$; subgroup 4 vs. 2 , $\mathrm{P}<0.001$; subgroup 4 vs. $3, \mathrm{P}=0.022$; subgroup 1 vs. $2, \mathrm{P}=0.919$; subgroup 1 vs. 3 , $\mathrm{P}=0.136$; subgroup 2 vs. 3 , $\mathrm{P}=0.213$ ). Subgroup 1, preoperative serum CA125 $\leq 15 \mathrm{U} / \mathrm{ml}$ and preoperative serum AFP $\leq 100 \mathrm{ng} / \mathrm{ml}(\mathrm{n}=95)$; subgroup 2, preoperative serum CA125 $\leq 15 \mathrm{U} / \mathrm{ml}$ and preoperative serum AFP $>100 \mathrm{ng} / \mathrm{ml}(\mathrm{n}=75)$; subgroup 3, preoperative serum CA125 $>15 \mathrm{U} / \mathrm{ml}$ and preoperative serum AFP $\leq 100 \mathrm{ng} / \mathrm{ml}$ ( $\mathrm{n}=57$ ); subgroup 4 , preoperative serum CA125 >15 U/ml and preoperative serum AFP $\geq 100 \mathrm{ng} / \mathrm{ml}(\mathrm{n}=79)$. CA125, cancer antigen 125; AFP, $\alpha$-fetoprotein; CI, confidence interval.

factors as significantly associated with OS: Diabetes, tumor size, tumor number, venous invasion, tumor differentiation and intraoperation transfusion. The results of the multivariate analysis demonstrated that tumor size, venous invasion and intraoperative transfusion were independent risk factors for OS (Table V). In the DFS analysis, no significant differences were observed in the DFS rates between the high and low preoperative serum CA125 groups in patients with low baseline AFP levels (subgroup 1 vs. 3, $\mathrm{P}=0.743$; Fig. 4A). Both univariate and multivariate analyses demonstrated that intraoperative transfusion and tumor size were prognostic factors associated with DFS and OS in these subgroups (Table V).

Patients with high preoperative serum CA125 and AFP levels have a poor prognosis. Kaplan-Meier analysis demonstrated that subgroup 4 had a significantly lower DFS rate compared with that in the other subgroups (subgroup 4 vs. $1, \mathrm{P}<0.001$; subgroup 4 vs. 2, $\mathrm{P}<0.001$; and subgroup 4 vs. $3, \mathrm{P}=0.009$ ). Among subgroups 1, 2 and 3, the differences were not significant (Fig. 4). In addition, patients in subgroup 4 had a significantly lower OS rate compared with that in the other three subgroups (subgroup 4 vs. 1, $\mathrm{P}<0.001$; subgroup 4 vs. 2, $\mathrm{P}<0.001$; and subgroup 4 vs. $3, \mathrm{P}=0.022)$. Comparisons between subgroups 2 and 3 and between subgroups 1 and 2 revealed no significant differences (Fig. 4).

\section{Discussion}

The key result of the present study was the identification of high preoperative serum CA125 levels as an independent risk factor for the prognosis of patients with HBV-related HCC. In addition, patients with both high preoperative serum CA125 and AFP levels presented with a poorer prognosis compared with that of patients with only high preoperative serum CA125 or AFP levels. However, no significant differences were observed in the DFS or OS rates between the high and low preoperative serum CA125 level groups in patients with baseline preoperative serum AFP levels $\leq 100 \mathrm{ng} / \mathrm{ml}$. Thus, the present study identified a new approach to predict the prognosis of patients with $\mathrm{HBV}$-related $\mathrm{HCC}$ patients using preoperative serum AFP and CA125 concentrations. This may provide a novel potential strategy to predict the prognosis and assess the course of treatment in patients with HBV-related HCC.

CA125 has been extensively used as a biomarker for ovarian cancer, and its upregulation has been observed in several types of human malignancy, such as lung, breast and pancreatic cancer (27-29). The normal reference value of CA125 is 0.1-35 U/ml (12); however, the $35 \mathrm{U} / \mathrm{ml}$ cutoff is not absolute. In one study, the serum CA125 concentration in healthy subjects was $7.9 \pm 8.0$ (mean $\pm \mathrm{SD}$ ) in women and $8.0 \pm 9.4 \mathrm{U} / \mathrm{ml}$ in men (12). Another study reported that the concentration was $13.2 \pm 6.8$ and $9.7 \pm 3.2 \mathrm{U} / \mathrm{ml}$ in women and men, respectively (30). With the benchmark cutoff of $35 \mathrm{U} / \mathrm{ml}$, $28 \%$ of patients with non-gynecological cancers had elevated antigen levels; by contrast, antigen levels were elevated in $>80 \%$ of women with non-mucinous ovarian cancer (12). Lopez et al (19) demonstrated that the CA125 cutoff values for patients with $\mathrm{HCC}$ were $55 \mathrm{U} / \mathrm{ml}$ in women and $12 \mathrm{U} / \mathrm{ml}$ in men. To date, the major focus of studies on CA125 levels and HCC was on the diagnosis, and only a limited number of studies have focused on the prognosis of HCC $(13,15,24)$. In addition, the association between CA125 and prognosis in patients with HBV-related HCC has not been widely reported. The present study used a CA125 cutoff value of $15 \mathrm{U} / \mathrm{ml}$ to investigate the association between preoperative serum CA125 levels and the prognosis of patients with HBV-related HCC.

In the present study, patients with high preoperative serum CA125 levels had significantly higher DFS and OS rates compared with those with low serum CA125 levels in all study populations. Zhou et al (24) have reported that high preoperative serum CA125 levels predict a poor prognosis and large tumor sizes following liver resection in patients with HCC and AFP 
Table V. Univariate and multivariate analyses of DFS and OS in patients with high preoperative serum AFP $\leq 100 \mathrm{ng} / \mathrm{ml}(\mathrm{n}=152)$.

\begin{tabular}{|c|c|c|c|c|c|c|c|c|}
\hline \multirow[b]{3}{*}{ Index } & \multicolumn{4}{|c|}{ DFS } & \multicolumn{4}{|c|}{ OS } \\
\hline & \multicolumn{2}{|c|}{$\begin{array}{l}\text { Univariate } \\
\text { analysis }\end{array}$} & \multicolumn{2}{|c|}{ Multivariate analysis } & \multicolumn{2}{|c|}{$\begin{array}{l}\text { Univariate } \\
\text { analysis }\end{array}$} & \multicolumn{2}{|c|}{ Multivariate analysis } \\
\hline & HR & P-value & HR $(95 \% \mathrm{CI})$ & P-value & HR & $\mathrm{P}$-value & HR $(95 \% \mathrm{CI})$ & $\mathrm{P}$-value \\
\hline Age (years) & 1.020 & 0.943 & & & 1.284 & 0.439 & & \\
\hline $\operatorname{Sex}(M ; n, \%)$ & 0.458 & 0.049 & & & 0.390 & 0.071 & & \\
\hline Hypertension & 1.214 & 0.551 & & & 0.798 & 0.540 & & \\
\hline Diabetes & 1.512 & 0.095 & & & 1.855 & 0.039 & & \\
\hline ALT & 1.273 & 0.297 & & & 0.945 & 0.841 & & \\
\hline AST & 1.005 & 0.984 & & & 0.725 & 0.277 & & \\
\hline Platelet count ( $\geq 100$ vs. $\left.<100 \times 10^{9} / 1\right)$ & 0.491 & 0.166 & & & 0.698 & 0.547 & & \\
\hline PT $(\leq 14$ vs. $>14 \mathrm{sec})$ & 1.214 & 0.431 & & & 0.911 & 0.756 & & \\
\hline TBIL ( $\leq 17$ vs. $>17 \mu \mathrm{mol} / \mathrm{l})$ & 1.126 & 0.624 & & & 1.049 & 0.878 & & \\
\hline $\operatorname{HBV}$ DNA $(\leq 2,000$ vs. $>2,000 \mathrm{IU} / \mathrm{ml})$ & 1.106 & 0.668 & & & 1.110 & 0.724 & & \\
\hline \multicolumn{9}{|l|}{ TNM stage } \\
\hline I & (Ref.) & (Ref.) & & & (Ref.) & (Ref.) & & \\
\hline II & 1.576 & 0.103 & & & 1.331 & 0.433 & & \\
\hline III & 1.280 & 0.373 & & & 1.700 & 0.107 & & \\
\hline \multicolumn{9}{|l|}{ ASA } \\
\hline I & (Ref.) & (Ref.) & & & (Ref.) & (Ref.) & & \\
\hline II & 1.106 & 0.676 & & & 0.809 & 0.729 & & \\
\hline III & 2.210 & 0.053 & & & 1.080 & 0.902 & & \\
\hline Child-Pugh grade (A vs. B) & 1.033 & 0.909 & & & 0.841 & 0.638 & & \\
\hline Cirrhosis & 0.911 & 0.753 & & & 1.313 & 0.505 & & \\
\hline Ascites & 1.336 & 0.237 & & & 1.402 & 0.258 & & \\
\hline Tumor diameter $(\leq 5 \mathrm{vs} .>5 \mathrm{~cm})$ & 2.305 & $<0.001$ & $\begin{array}{c}1.945 \\
(1.206-3.137)\end{array}$ & 0.006 & 2.527 & 0.003 & $\begin{array}{c}2.043 \\
(1.091-3.825)\end{array}$ & 0.026 \\
\hline Tumor number (Multiple vs. solitary) & 2.270 & 0.001 & 2.099 & 0.003 & 1.924 & 0.029 & & \\
\hline Venous invasion (Yes vs. no) & 1.673 & 0.029 & $(1.298-3.396)$ & & 2.261 & 0.004 & $\begin{array}{c}2.016 \\
(1.142-3.559)\end{array}$ & 0.016 \\
\hline \multicolumn{9}{|l|}{ Tumor differentiation } \\
\hline Well differentiated & (Ref.) & (Ref.) & & & (Ref.) & (Ref.) & & \\
\hline Moderately differentiated & 2.181 & 0.029 & & & 4.309 & 0.015 & & \\
\hline Poorly differentiated & 1.770 & 0.344 & & & 3.989 & 0.093 & & \\
\hline $\begin{array}{l}\text { Intraoperative blood loss } \\
(\leq 1,000 \text { vs. }>1,000 \mathrm{ml})\end{array}$ & 1.547 & 0.101 & & & 1.851 & 0.056 & & \\
\hline Intraoperative transfusion (Yes vs. no) & 2.047 & 0.002 & $\begin{array}{c}1.916 \\
(1.208-3.040)\end{array}$ & 0.006 & 2.184 & 0.006 & $\begin{array}{c}1.932 \\
(1.091-3.419)\end{array}$ & 0.024 \\
\hline TACE ( $\leq 3$ vs. $>3$ ) & 1.387 & 0.254 & & & 0.935 & 0.862 & & \\
\hline CA125 ( $\leq 15$ vs. $>15 \mathrm{U} / \mathrm{ml})$ & 1.093 & 0.703 & & & 1.557 & 0.119 & & \\
\hline
\end{tabular}

DFS, disease-free survival; OS, overall survival; HBV, hepatitis B virus; ASA, American Society of Anesthesiologists; TNM, Tumor-Node-Metastasis; ALT, alanine aminotransferase; AST, aspartate aminotransferase; PT, prothrombin time; TBIL, serum total bilirubin; AFP, $\alpha$-fetoprotein; TACE, transcatheter arterial chemoembolization; CA125, cancer antigen 125; HR, hazard ratio; CI, confidence interval.

$\leq 200 \mathrm{ng} / \mathrm{ml}$. However, the aforementioned study did not identify high preoperative serum CA125 levels as an independent risk factor for the prognosis of HCC. Although Huang et al (13) have demonstrated that high preoperative serum CA125 levels served as an independent prognostic factor of OS and DFS in patients with HCC, the cutoff values for CA125 and AFP levels in their study were $35 \mathrm{U} / \mathrm{ml}$ and $20 \mathrm{ng} / \mathrm{ml}$, respectively. On the other hand, the 3 -year overall survival rates in the normal and high CA125 HCC groups in their study were 53.6 and $36.4 \%$, respectively (13), which were slightly lower compared with those observed in the present study. The main reason for these differences may be the inclusion of patients with non-HBV-related 
HCC in the previous study. In the present study, the results of the multivariate analysis demonstrated that tumor diameter, multiple tumors and venous invasion were independent risk factors for DFS and OS, and a high preoperative serum CA125 level was an independent risk factor associated with a short OS time in patients with HBV-related HCC.

In the stratification analyses in the present study, a low preoperative serum CA125 level was associated with a favorable prognosis (both OS and DFS rates) in patients with high baseline AFP levels; the same was observed in patients with low preoperative serum AFP and high baseline CA125 levels. However, in patients with low baseline AFP levels, no significant differences were observed in the DFS or OS rates between the preoperative high and low serum CA125 level subgroups. A notable outcome of the present study was that patients with high preoperative serum CA125 and AFP levels presented with a poorer prognosis compared with that in the other three groups. Certain tumor characteristics of subgroup 4 (TNM stage III, tumor diameter, multiple tumors, presence of venous invasion and tumor differentiation) were associated with higher malignancy compared with those in the other groups, which may be due to the patients with both high preoperative serum CA125 and AFP levels exhibiting a tendency for a poor prognosis. A previous study has demonstrated that high serum AFP levels tend to indicate highly malignant tumors with histological features of aggressiveness such as poor differentiation, vascular invasion, satellitosis and a fast growth rate (31). High serum CA125 levels predict a large tumor diameter (24), which was observed in the present study in patients with the same baseline AFP levels. In the stratification analyses, multivariate analysis demonstrated that tumor diameter, multiple tumors and the presence of venous invasion were independent risk factors for DFS and OS. Based on low preoperative serum AFP and CA125 levels, multivariate analysis demonstrated that intraoperative transfusion was an independent risk factor for DFS and OS. One previous meta-analysis has demonstrated that perioperative blood transfusion is associated with adverse clinical outcomes in patients following resection of HCC (32). The explanation proposed by the authors of the aforementioned study is that allogenic blood transfusion may induce immunosuppression and decrease natural killer (NK) cell and/or $\mathrm{T}$ helper cell activities. Thus, the prognosis of patients with HBV-related HCC may be associated not only with the biological characteristics of the tumor, but also with the perioperative management of patients.

However, further studies are required to determine the underlying mechanism of the effects of CA125 in tumor development. Previous studies have demonstrated that upregulation of CA125 leads to tumor growth and metastasis (33-35), which may explain the poor prognosis of patients with HBV-related HCC. The MUC16 gene, which is also termed CA125, has been identified to be one of the top three frequently mutated genes (36), and its upregulation has been associated with a poor prognosis in multiple types of malignancy, such as pancreatic cancer, cholangiocarcinoma and bladder cancer $(21,37-39)$. CA125 has been demonstrated to modulate the innate immune response against ovarian cancer cells by directly inhibiting the function of NK cells, thus aiding cancer cells to escape the host immune response $(40,41)$. In addition, MUC16 has been implicated in cancer cell signaling; knockdown of $M U C 16$ expression inhibits the proliferation of ovarian and breast cancer cell lines by inducing caspase-dependent or independent apoptosis (42). Furthermore, the aforementioned study has also reported that $M U C 16$ knockdown suppresses the colony-forming, adhesive, migratory and invasive abilities of ovarian and breast cancer cells. Thus, CA125 may become a novel target for the treatment of HBV-related HCC.

The present study identified that patients with high preoperative serum AFP levels were younger compared with those with low preoperative serum AFP levels, which was consistent with the results of a previous study (43). In addition, in the present study, patients with the same baseline AFP levels but with high serum CA125 levels presented with poorer liver function compared with that in patients with low serum CA125 levels, whereas no significant differences were observed in the preoperative liver function between the preoperative high and low serum AFP level groups when the baseline CA125 levels were the same. These results suggested that patients with high CA125 levels may have a longer liver disease history. Previous studies have reported that high levels of CA 125 were associated with the severity of liver disease, particularly in patients with cirrhosis $(17,44,45)$, which is a late stage of liver disease; however, this should be validated in a prospective randomized controlled study by monitoring the dynamic levels of CA125. Therefore, the results of the current study should be interpreted carefully.

The present study had various limitations. First, the study utilized a retrospective design, and the patient sample size was relatively small following stratification. Second, a subset of patients who underwent surgery in 2018 was included; therefore, the follow-up was not sufficiently long, which potentially affected the survival analysis outcome. Third, the effect of abdominal inflammation before surgery could not be ruled out, and an increase in CA125 levels may be associated with peritoneal inflammation (17). Fourth, the dynamic changes in CA125 levels after surgery were not monitored, and whether high postoperative serum CA125 level is a risk factor for poor prognosis in patients with $\mathrm{HBV}$-related $\mathrm{HCC}$ following resection is unknown. In addition, in the OS curves, a small gap was observed between subgroups 1 and 3 , although no significant difference existed; thus, a longer follow-up period and a larger number of cases is required to address this issue. The results of the present study need to be validated further in prospective randomized large-sample multicenter studies.

In conclusion, the present study used a $15 \mathrm{U} / \mathrm{ml}$ cutoff value of CA125 and demonstrated that preoperative serum CA125 levels may be a prognostic factor for survival in patients with HBV-related HCC. However, in patients with low AFP levels, the association between preoperative serum CA125 levels and prognosis in HBV-related HCC remains unclear and requires further investigation.

\section{Acknowledgements}

The authors would like to thank Mr. Guangdou Yuan (The First Affiliated Hospital of Guangxi Medical University, Nanning, China) for his technical support. 


\section{Funding}

This study was supported by The Liuzhou Medical Basic Research Foundation (grant no. 1ry201705) and the Self-funded Project of Guangxi Health Commission (grant no. Z20170685).

\section{Availability of data and materials}

The datasets used and/or analyzed during the current study are available from the corresponding author on reasonable request.

\section{Authors' contributions}

CQ and SH conceived and designed the current study. CQ wrote and revised the manuscript. SH performed the majority of the experiments. YG participated in the research design, the collection, sorting and statistical analysis of research data, and helped to edit the manuscript. $\mathrm{SH}$ and $\mathrm{CH}$ confirmed the authenticity of all the raw data and analyzed statistical data. $\mathrm{CH}$ and JL interpreted the data and revised the manuscript. All authors read and approved the final version of the manuscript.

\section{Ethics approval and consent to participate}

The study complied with the standards of the declaration of Helsinki and was approved by The Clinical Research Ethics Committee of Liuzhou People's Hospital Affiliated to Guangxi Medical University (approval no. 2020-KY-E-13-01). The need for informed consent was waived owing to the retrospective nature of the study.

\section{Patient consent for publication}

Not applicable.

\section{Competing interests}

The authors declare that they have no competing interests.

\section{References}

1. Siegel RL, Miller KD and Jemal A: Cancer statistics, 2020. CA Cancer J Clin 70: 7-30, 2020.

2. Bray F, Ferlay J, Soerjomataram I, Siegel RL, Torre LA and Jemal A: Global cancer statistics 2018: GLOBOCAN estimates of incidence and mortality worldwide for 36 cancers in 185 countries. CA Cancer J Clin 68: 394-424, 2018.

3. Zamor PJ, deLemos AS and Russo MW: Viral hepatitis and hepatocellular carcinoma: Etiology and management. J Gastrointest Oncol 8: 229-242, 2017.

4. Nguyen VT, Law MG and Dore GJ: Hepatitis B-related hepatocellular carcinoma: Epidemiological characteristics and disease burden. J Viral Hepat 16: 453-463, 2009.

5. Bruix J, Gores GJ and Mazzaferro V: Hepatocellular carcinoma: Clinical frontiers and perspectives. Gut 63: 844-855, 2014.

6. Miura JT, Johnston FM, Tsai S, Eastwood D, Banerjee A, Christians KK, Turaga KK and Gamblin TC: Surgical resection versus ablation for hepatocellular carcinoma $</=3 \mathrm{~cm}$ : A population-based analysis. HPB (Oxford) 17: 896-901, 2015.

7. Rahbari NN, Mehrabi A, Mollberg NM, Müller SA, Koch M, Büchler MW and Weitz J: Hepatocellular carcinoma: Current management and perspectives for the future. Ann Surg 253, 453-469, 2011.
8. Akamatsu $\mathrm{N}$ and Kokudo $\mathrm{N}$ : Liver transplantation for hepatocellular carcinoma from living-donor vs. Deceased donor. Hepatobiliary Surg Nutr 5: 422-428, 2016.

9. Hyun MH, Lee YS, Kim JH, Lee CU, Jung YK, Seo YS, Yim HJ, Yeon JE and Byun KS: Hepatic resection compared to chemoembolization in intermediate- to advanced-stage hepatocellular carcinoma: A meta-analysis of high-quality studies. Hepatology 68: 977-993, 2018.

10. Trevisani F, Bucci L and Garuti F: Is it time to extend criteria for hepatic resection in the treatment of hepatocellular carcinoma? Hepatology 64: 2257-2258, 2016.

11. Bast RC Jr, Feeney M, Lazarus H, Nadler LM, Colvin RB and Knapp RC: Reactivity of a monoclonal antibody with human ovarian carcinoma. J Clin Invest 68: 1331-1337, 1981.

12. Bast RC Jr, Klug TL, St John E, Jenison E, Niloff JM, Lazarus H, Berkowitz RS, Leavitt T, Griffiths CT, Parker L, et al: A radioimmunoassay using a monoclonal antibody to monitor the course of epithelial ovarian cancer. N Engl J Med 309: 883-887, 1983.

13. Huang Y, Zeng J, Liu T, Lin X, Guo P, Zeng J, Zhou W and Liu J: Prognostic significance of elevated preoperative serum CA125 levels after curative hepatectomy for hepatocellular carcinoma. Onco Targets Ther 13: 4559-4567, 2020.

14. Chen K, Gentry-Maharaj A, Burnell M, Steentoft C, Marcos-Silva L, Mandel U, Jacobs I, Dawnay A, Menon U and Blixt O: Microarray Glycoprofiling of CA125 improves differential diagnosis of ovarian cancer. J Proteome Res 12: 1408-1418, 2013.

15. Li Y, Li DJ, Chen J, Liu W, Li JW, Jiang P, Zhao X, Guo F, Li XW and Wang SG: Application of Joint Detection of AFP, CA19-9, CA125 and CEA in identification and diagnosis of cholangiocarcinoma. Asian Pac J Cancer Prev 16: 3451-3455, 2015.

16. Wu LX, Li XF, Chen HF, Zhu YC, Wang WX, Xu CW, Xie DF, Wan Y and Du KQ: Combined detection of CEA and CA125 for the diagnosis for lung cancer: A meta-analysis. Cell Mol Biol (Noisy-le-grand) 64: 67-70, 2018.

17. Devarbhavi H, Kaese D, Williams AW, Rakela J, Klee GG and Kamath PS: Cancer antigen 125 in patients with chronic liver disease. Mayo Clin Proc 77: 538-541, 2002.

18. Bergmann JF, Bidart JM, George M, Beaugrand M, Levy VG and Bohuon C: Elevation of CA 125 in patients with benign and malignant ascites. Cancer 59: 213-217, 1987.

19. Lopez JB, Balasegaram M and Thambyrajah V: Serum CA 125 as a marker of hepatocellular carcinoma. Int J Biol Markers 11: 178-182, 1996.

20. Piñero F, Dirchwolf M and Pessôa MG: Biomarkers in hepatocellular carcinoma: Diagnosis, prognosis and treatment response assessment. Cells 9: 1370, 2020.

21. Liang C, Qin Y, Zhang B, Ji S, Shi S, Xu W, Liu J, Xiang J, Liang D, Hu Q, et al: Oncogenic KRAS Targets MUC16/CA125 in pancreatic ductal adenocarcinoma. Mol Cancer Res 15: 201-212, 2017.

22. Pradjatmo $\mathrm{H}$ and Pradjatmo $\mathrm{H}$ : Impact of preoperative serum levels of CA 125 on epithelial ovarian cancer survival. Asian Pac J Cancer Prev 17: 1881-1886, 2016.

23. Isaksson $\mathrm{S}$, Jönsson $\mathrm{P}$, Monsef $\mathrm{N}$, Brunnström H, Bendahl PO, Jönsson M, Staaf J and Planck M: CA 19-9 and CA 125 as potential predictors of disease recurrence in resectable lung adenocarcinoma. PLoS One 12: e0186284, 2017.

24. Zhou S, Wang Z, Li M and Wu L: Elevated preoperative serum CA125 predicts larger tumor diameter in patients with hepatocellular carcinoma and low AFP levels. Biomed Res Int 2019: 6959637, 2019.

25. Chong CC, Lee KF, Ip PC, Wong JS, Cheung SY, Wong J, Ho SC and Lai PB: Pre-operative predictors of post-hepatectomy recurrence of hepatocellular carcinoma: Can we predict earlier? Surgeon 10: 260-266, 2012.

26. Terrault NA, Bzowej NH, Chang KM, Hwang JP, Jonas MM and Murad MH; American Association for the Study of Liver Diseases: AASLD guidelines for treatment of chronic hepatitis B. Hepatology 63: 261-283, 2016.

27. Haridas D, Chakraborty S, Ponnusamy MP, Lakshmanan I, Rachagani S, CruzE, Kumar S, Das S, Lele SM, Anderson JM, et al: Pathobiological implications of MUC16 expression in pancreatic cancer. PLoS One 6: e26839, 2011.

28. Lakshmanan I, Ponnusamy MP, Das S, Chakraborty S, Haridas D, Mukhopadhyay P, Lele SM and Batra SK: MUC16 induced rapid $\mathrm{G} 2 / \mathrm{M}$ transition via interactions with JAK2 for increased proliferation and anti-apoptosis in breast cancer cells. Oncogene 31: 805-817, 2012. 
29. Lakshmanan I, Salfity S, Seshacharyulu P, Rachagani S, Thomas A, Das S, Majhi PD, Nimmakayala RK, Vengoji R, Lele SM, et al: MUC16 Regulates TSPYL5 for lung cancer cell growth and chemoresistance by suppressing p53. Clin Cancer Res 23: 3906-3917, 2017.

30. Klug TL, Bast RC Jr, Niloff JM, Knapp RC and Zurawski VR Jr: Monoclonal antibody immunoradiometric assay for an antigenic determinant (CA 125) associated with human epithelial ovarian carcinomas. Cancer Res 44: 1048-1053, 1984.

31. Trevisani F, Garuti F and Neri A: Alpha-fetoprotein for diagnosis, prognosis, and transplant selection. Semin Liver Dis 39: $163-177,2019$.

32. Liu L, Wang Z, Jiang S, Shao B, Liu J, Zhang S, Zhou Y, Zhou Y and Zhang Y: Perioperative allogenenic blood transfusion is associated with worse clinical outcomes for hepatocellular carcinoma: A meta-analysis. PLoS One 8: e64261, 2013.

33. Rao TD, Tian H, Ma X, Yan X, Thapi S, Schultz N, Rosales N, Monette S, Wang A, Hyman DM, et al: Expression of the carboxy-terminal portion of MUC16/CA125 induces transformation and tumor invasion. PLoS One 10: e0126633, 2015.

34. Das S, Rachagani S, Torres-Gonzalez MP, Lakshmanan I, Majhi PD, Smith LM, Wagner KU and Batra SK: Carboxyl-terminal domain of MUC16 imparts tumorigenic and metastatic functions through nuclear translocation of JAK2 to pancreatic cancer cells. Oncotarget 6: 5772-5787, 2015.

35. Giannakouros P, Matte I, Rancourt C and Piche A: Transformation of NIH3T3 mouse fibroblast cells by MUC16 mucin (CA125) is driven by its cytoplasmic tail. Int J Oncol 46: 91-98, 2015.

36. Kim N, Hong Y, Kwon D and Yoon S: Somatic mutaome profile in human cancer tissues. Genomics Inform 11: 239-244, 2013.

37. Chen SH, Hung WC, Wang P, Paul C and Konstantopoulos K: Mesothelin binding to CA125/MUC16 promotes pancreatic cancer cell motility and invasion via MMP-7 activation. Sci Rep 3: 1870, 2013

38. Higashi M, Yamada N, Yokoyama S, Kitamoto S, Tabata K, Koriyama C, Batra SK and Yonezawa S: Pathobiological implications of MUC16/CA125 expression in intrahepatic cholangiocarcinoma-mass forming type. Pathobiology 79: 101-116, 2012.
39. Cotton S, Azevedo R, Gaiteiro C, Ferreira D, Lima L, Peixoto A, Fernandes E, Neves M, Neves D, Amaro T, et al: Targeted $\mathrm{O}$-glycoproteomics explored increased sialylation and identified MUC16 as a poor prognosis biomarker in advanced-stage bladder tumours. Mol Oncol 11: 895-912, 2017.

40. Belisle JA, Horibata S, Jennifer GA, Petrie S, Kapur A, André S, Gabius HJ, Rancourt C, Connor J, Paulson JC and Patankar MS: Identification of Siglec-9 as the receptor for MUC16 on human NK cells, B cells, and monocytes. Mol Cancer 9: 118, 2010.

41. Gubbels JA, Felder M, Horibata S, Belisle JA, Kapur A, Holden H, Petrie S, Migneault M, Rancourt C, Connor JP and Patankar MS: MUC16 provides immune protection by inhibiting synapse formation between NK and ovarian tumor cells. Mol Cancer 9: 11, 2010.

42. Reinartz S, Failer S, Schuell T and Wagner U: CA125 (MUC16) gene silencing suppresses growth properties of ovarian and breast cancer cells. Eur J Cancer 48: 1558-1569, 2012.

43. Peng SY, Chen WJ, Lai PL, Jeng YM, Sheu JC and Hsu HC: High alpha-fetoprotein level correlates with high stage, early recurrence and poor prognosis of hepatocellular carcinoma: Significance of hepatitis virus infection, age, p53 and beta-catenin mutations. Int J Cancer 112: 44-50, 2004.

44. Edula RG, Muthukuru S, Moroianu S, Wang Y, Lingiah V, Fung P and Pyrsopoulos NT: CA-125 significance in cirrhosis and correlation with disease severity and portal hypertension: A retrospective study. J Clin Transl Hepatol 6: 241-246, 2018.

45. Singhal A, Lander E, Karachristos A, Daly E, Dowling P, Patel V, Maloo M and Jain A: Elevation of CA 125 and CA 19-9 in patients with end-stage liver disease. Int J Biol Markers 27: e147-e151, 2012

This work is licensed under a Creative Commons Attribution-NonCommercial-NoDerivatives 4.0 International (CC BY-NC-ND 4.0) License. 